Research Article

\title{
Network Pharmacology-Based Prediction of Mechanism of Shenzhuo Formula for Application to DKD
}

\author{
Xinmiao Wang $\mathbb{D}^{1},{ }^{1}$ Haoyu Yang $\mathbb{D},{ }^{1,2}$ Lili Zhang $\mathbb{D}^{1},{ }^{1}$ Lin Han, ${ }^{1}$ Sha Di, ${ }^{1}$ Xiuxiu Wei, ${ }^{1,2}$ \\ Haoran Wu, ${ }^{1,2}$ Haiyu Zhang, ${ }^{1,2}$ Linhua Zhao $\mathbb{D}^{3},{ }^{3}$ and Xiaolin Tong $\mathbb{D}^{4}$ \\ ${ }^{1}$ Department of Endocrinology, Guang'anmen Hospital, China Academy of Chinese Medical Sciences, Beijing 100053, China \\ ${ }^{2}$ Graduate College, Beijing University of Traditional Chinese Medicine, Beijing 100029, China \\ ${ }^{3}$ Laboratory of Molecular and Biology, Guang'anmen Hospital, China Academy of Chinese Medical Sciences, \\ Beijing 100053, China \\ ${ }^{4}$ Department of Endocrinology, Affiliated Hospital to Changchun University of Chinese Medicine, Changchun 130021, China
}

Correspondence should be addressed to Linhua Zhao; melonzhao@163.com and Xiaolin Tong; tongxiaolin@vip.163.com

Received 3 October 2020; Revised 19 February 2021; Accepted 12 April 2021; Published 21 April 2021

Academic Editor: Daniel Dias Rufino Arcanjo

Copyright ( $\odot 2021$ Xinmiao Wang et al. This is an open access article distributed under the Creative Commons Attribution License, which permits unrestricted use, distribution, and reproduction in any medium, provided the original work is properly cited.

\begin{abstract}
Background. Shenzhuo formula (SZF) is a traditional Chinese medicine (TCM) prescription which has significant therapeutic effects on diabetic kidney disease (DKD). However, its mechanism remains unknown. Therefore, this study aimed to explore the underlying anti-DKD mechanism of SZF. Methods. The active ingredients and targets of SZF were obtained by searching TCMSP, TCMID, SwissTargetPrediction, HIT, and literature. The DKD target was identified from TTD, DrugBank, and DisGeNet. The potential targets were obtained and PPI network were built after mapping SZF targets and DKD targets. The key targets were screened out by network topology and the "SZF-key targets-DKD" network was constructed by Cytoscape. GO analysis and KEGG pathway enrichment analysis were performed by using DAVID, and the results were visualized by Omicshare Tools. Results. We obtained 182 potential targets and 30 key targets. Furthermore, a "SZF-key targets-DKD" network topological analysis showed that active ingredients like M51, M21, M5, M71, and M28 and targets like EGFR, MMP9, MAPK8, PIK3CA, and STAT3 might play important roles in the process of SZF treating in DKD. GO analysis results showed that targets were mainly involved in positive regulation of transcription from RNA polymerase II promoter, inflammatory response, lipopolysaccharide-mediated signaling pathway, and other biological processes. KEGG showed that DKD-related pathways like TNF signaling pathway and PI3K-Akt signaling pathway were at the top of the list. Conclusion. This research reveals the potential pharmacological targets of SZF in the treatment of DKD through network pharmacology and lays a foundation for further studies.
\end{abstract}

\section{Introduction}

Diabetic kidney disease (DKD) is one of the most common chronic microvascular complications of diabetes. It may be caused and shaped by the interaction of many factors such as endoplasmic reticulum dysfunction, high sugar-mediated generation of terminal advanced glycation endproducts (AGE), increased activation of the renin angiotensin aldosterone system, increased generation of reactive oxygen species (ROS), and activation of extracellular matrix (ECM) and protein kinase $C[1,2]$. It is reported that the incidence of DKD is about $40 \%$ in the diabetic population [3].
Furthermore, with the increasing incidence of diabetes, the incidence of DKD is increasing yearly [4]. Therefore, it is important to intensify studies of the pathogenesis of DKD and the search for effective intervention targets.

Shenzhuo formula (SZF) as a traditional Chinese medicine (TCM) prescription has certain advantages in the treatment of DKD [5].It is created by Tong Xiaolin, an academician at the Chinese Academy of Sciences, and his team. This formula was based on the pathogenesis of qi deficiency blood stasis, and the classic prescription of Didang decoction. Years of clinical studies have shown that SZF can effectively increase the glomerular filtration rate, 
reduce 24-hour urinary protein and kidney damage, and reverse kidney disease when used early $[5,6]$. However, due to the diversity of TCM compounds and complexity of in vivo processes, the systematic mechanism research of SZF has been hindered.

Recently, network pharmacology has been developed rapidly with the use of multiomics, high-throughput screening, network visualization and analysis, or other techniques [7-9]. It can help to reveal the network structure of drug action [10] and provide possibilities for exploring the mechanism of action of TCM compounds. Therefore, this study aimed to shed light on the underlying mechanisms of SZF in DKD treatment using a network pharmacology approach.

\section{Methods}

2.1. Research Tools. The Chinese Traditional Medicine System Pharmacological Database Analysis Platform (TCMSP, http:// lsp.nwu.edu.cn/tcmsp.php) [11], Traditional Chinese Medicine Integrated Database (TCMID, http://www.megabionet.org/ tcmid/) [12], SwissTargetPrediction (http://www.swisstargetpred iction.ch/) [13], and HIT (http:lifecenter.biosino.org/hit/) [14] were used to access to SZF ingredients and targets. (2) The Therapeutic Target Database (TTD, http://bidd.nus.edu.sg/ group/cjttd/) [15], DrugBank (https://www.drugbank.ca/) [16], and DisGeNet (http://www.disgenet.org/) [17] were used to get the targets' proteins of DKD. (3) The protein-protein interaction (PPI) network was obtained online using STRING (http:// string-db.org) [18]. Compositional software Cytoscape 3.2.1 (http://www.cytoscape.org/) [19] was used to carry out network topology analysis and construct SZF-key targets-DKD network. The Database for Annotation, Visualization and Integrated Discovery (DAVID, http://david.ncifcrf.Gov) [20] was used for Gene Ontology (GO) and Kyoto Encyclopedia of Genes and Genomes (KEGG) analysis. The Omicshare Tools (https://www. Omicshare.com/) were used for visual analysis of GO and KEGG results.

2.2. Collection of Major Chemical Constituents. We relied on TCMSP, TCMID database, and literatures mining to search for the chemical constituents of SZF (Hedysarum Multijugum Maxim, Radix Salviae, Hirudo, and Radix Rhei Et Rhizome).

2.3. Screening of Active Compounds. As we all know, TCM drugs enter human body and then take effect through absorption, distribution, metabolism, and excretion (ADME) processes. Among them, oral bioavailability (OB) and drug similarity (DL), the key parameters of ADME components, were used as the screening criteria for active ingredients in this study. In this section, we used TCMSP to collect active compounds and their ADME properties. And then the active compounds that meet " $\mathrm{OB} \geq 30 \%, \mathrm{DL} \geq 0.18$ " were selected as potential active ingredients.

2.4. Prediction of Targets. SwissTargetPrediction and HIT databases were used to collect the drug targets. In addition, TTD, DrugBank and DisGeNet databases were used to search for DKD targets by entering the key words of "diabetic kidney disease" and "diabetic nephropathy." Further, we matched SZF targets with DKD targets to obtain common targets.

2.5. Network Construction and Analysis. PPI network of common targets was obtained using STRING. Furthermore, the PPI network topology analysis was carried out using Cytoscape 3.2.1 software and then key targets were obtained. To further explore the interactions between the active ingredients and their related targets at a system level, a "SZFkey targets-DKD" network was constructed by Cytoscape3.2.1.

2.6. GO and KEGG Analysis. GO analysis is widely used for gene function classification and mainly includes the molecular function (MF), biological processes (BP), and cellular components (CC) [21]. In this step, we used the DAVID tool for GO and KEGG pathway analysis. Then, we used Omicshare Tools for visual display.

\section{Results}

3.1. Screening of Candidate Components in SZF. Through TCMSP and TCMID database, a total of 87 active compounds of Hedysarum Multijugum Maxim, 210 of Radix Salviae, 35 of Hirudo, and 92 of Radix Rhei Et Rhizome were obtained. Then by ADME ( $\mathrm{OB} \geq 30 \%, \mathrm{DL} \geq 0.18)$ screening, a total of 101 active compounds were selected, including 20 active compounds of Hedysarum Multijugum Maxim, 65 of Radix Salviae, and 16 of Radix Rhei Et Rhizome (in this section, because Hirudo could not be found in TCMSP database, its ADME parameters could not be obtained and did not participate in screening). In addition, through literature mining, another 4 active compounds were collected, including 2 active compounds of Hedysarum Multijugum Maxim [22, 23], 1 of Radix Salviae [24], and 1 of Radix Rhei Et Rhizome [25].

3.2. Target Prediction. After matching SZF targets with DKD genes, a total of 182 common targets of SZF were obtained. We only show 50 of them in Table 1 . And full information of 182 common targets is displayed in Table 2.

3.3. Construction and Analysis of Network Maps. The PPI network of the 182 common targets was obtained using STRING (Figure 1). Then, we used Cytoscape 3.2.1 to obtain 30 key targets by network topology analysis with inclusion criteria of "degree $\geq 2$ times of the median, closeness centrality $\geq$ median, betweenness centrality $\geq$ median" (Table 3 ). Next, we constructed a "SZF-key targets-DKD" network by Cytoscape3.2.1 (Figure 2).

3.4. GO and KEGG Analysis. The DAVID was used to carry out GO analysis. And the GO terms were visualized by the Omicshare Tools (Figure 3). The GO analysis results showed that targets were mainly involved in positive regulation of 
TABLE 1: Common targets of SZF and DKD (50 of 182 targets).

\begin{tabular}{|c|c|c|c|}
\hline Serial number & Target & Common name & Uniprot ID \\
\hline 1 & Aldose reductase & AKR1B1 & P15121 \\
\hline 2 & Acyl coenzyme A:cholesterol acyltransferase & CES1 & P23141 \\
\hline 3 & Signal transducer and activator of transcription 3 & STAT3 & P40763 \\
\hline 4 & Protein-tyrosine phosphatase $1 \mathrm{C}$ & PTPN6 & P29350 \\
\hline 5 & Vascular endothelial growth factor receptor 2 & KDR & P35968 \\
\hline 6 & Epidermal growth factor receptor erbB1 & EGFR & P00533 \\
\hline 7 & PI3-kinase p110-alpha subunit & PIK3CA & P42336 \\
\hline 8 & c-Jun N-terminal kinase 1 & MAPK8 & P45983 \\
\hline 9 & LXR-alpha & NR1H3 & Q13133 \\
\hline 10 & Estrogen receptor alpha & ESR1 & P03372 \\
\hline 11 & Testis-specific androgen-binding protein & SHBG & P04278 \\
\hline 12 & Cytochrome P450 2C19 & CYP2C19 & P33261 \\
\hline 13 & Protein-tyrosine phosphatase $1 \mathrm{~B}$ & PTPN1 & P18031 \\
\hline 14 & Butyrylcholinesterase & BCHE & P06276 \\
\hline 15 & Vitamin D receptor & VDR & P11473 \\
\hline 16 & Glucose-6-phosphate 1-dehydrogenase & G6PD & P11413 \\
\hline 17 & Peroxisome proliferator-activated receptor alpha & PPARA & Q07869 \\
\hline 18 & Peroxisome proliferator-activated receptor delta & PPARD & Q03181 \\
\hline 19 & Peroxisome proliferator-activated receptor gamma & PPARG & P37231 \\
\hline 20 & UDP-glucuronosyltransferase $2 \mathrm{~B} 7$ & UGT2B7 & P16662 \\
\hline 21 & 11-Beta-hydroxysteroid dehydrogenase 2 & HSD11B2 & P80365 \\
\hline 22 & NADPH oxidase 4 & NOX4 & Q9NPH5 \\
\hline 23 & Tyrosine-protein kinase SYK & SYK & P43405 \\
\hline 24 & Glycogen synthase kinase- 3 beta & GSK3B & P49841 \\
\hline 25 & Matrix metalloproteinase 9 & MMP9 & P14780 \\
\hline 26 & Matrix metalloproteinase 2 & MMP2 & P08253 \\
\hline 27 & Matrix metalloproteinase 12 & MMP12 & P39900 \\
\hline 28 & ATP-binding cassette sub-family $\mathrm{G}$ member 2 & ABCG2 & Q9UNQ0 \\
\hline 29 & P-glycoprotein 1 & ABCB1 & P08183 \\
\hline 30 & Arachidonate 12-lipoxygenase & ALOX12 & P18054 \\
\hline 31 & Cyclooxygenase- 2 & PTGS2 & P35354 \\
\hline 32 & Insulin-like growth factor I receptor & IGF1R & P08069 \\
\hline 33 & Myeloperoxidase & MPO & P05164 \\
\hline 34 & Matrix metalloproteinase 3 & MMP3 & P08254 \\
\hline 35 & Serine/threonine-protein kinase AKT & AKT1 & P31749 \\
\hline 36 & Beta-secretase 1 & BACE1 & P56817 \\
\hline 37 & Tyrosine-protein kinase receptor UFO & AXL & P30530 \\
\hline 38 & NUAK family SNF1-like kinase 1 & NUAK1 & O60285 \\
\hline 39 & Aldehyde reductase & AKR1A1 & P14550 \\
\hline 40 & Plasminogen & PLG & P00747 \\
\hline 41 & PI3-kinase p110-delta subunit & PIK3CD & O00329 \\
\hline 42 & PI3-kinase p110-gamma subunit & PIK3CG & P48736 \\
\hline 43 & Hematopoietic prostaglandin D synthase & HPGDS & O60760 \\
\hline 44 & Serine-protein kinase ATM & ATM & Q13315 \\
\hline 45 & Cytochrome P450 24A1 & CYP24A1 & Q07973 \\
\hline 46 & Mineralocorticoid receptor & NR3C2 & P08235 \\
\hline 47 & Cannabinoid receptor 1 & CNR1 & P21554 \\
\hline 48 & Hepatocyte nuclear factor 4 -alpha & HNF4A & P41235 \\
\hline 49 & C-C chemokine receptor type 1 & CCR1 & P32246 \\
\hline 50 & Histone-lysine N-methyltransferase EZH2 & $\mathrm{EZH} 2$ & Q15910 \\
\hline
\end{tabular}

Organism: Homo sapiens. Only 50 potential targets' information is shown here, and the whole is in Table 3. 
TABLE 2: A total of 182 common targets.

\begin{tabular}{|c|c|c|c|}
\hline No. & Target & Common name & Uniprot ID \\
\hline 1 & Aldose reductase & AKR1B1 & P15121 \\
\hline 2 & Acyl coenzyme A:cholesterol acyltransferase & CES1 & P23141 \\
\hline 3 & Signal transducer and activator of transcription 3 & STAT3 & P40763 \\
\hline 4 & Protein-tyrosine phosphatase $1 \mathrm{C}$ & PTPN6 & P29350 \\
\hline 5 & Vascular endothelial growth factor receptor 2 & KDR & P35968 \\
\hline 6 & Epidermal growth factor receptor erbB1 & EGFR & P00533 \\
\hline 7 & PI3-kinase p110-alpha subunit & PIK3CA & P42336 \\
\hline 8 & c-Jun $\mathrm{N}$-terminal kinase 1 & MAPK8 & P45983 \\
\hline 9 & LXR-alpha & NR1H3 & Q13133 \\
\hline 10 & Estrogen receptor alpha & ESR1 & P03372 \\
\hline 11 & Testis-specific androgen-binding protein & SHBG & P04278 \\
\hline 12 & Cytochrome P450 2C19 13 & CYP2C19 & P33261 \\
\hline 13 & Protein-tyrosine phosphatase $1 \mathrm{~B}$ & PTPN1 & P18031 \\
\hline 14 & Butyrylcholinesterase & BCHE & P06276 \\
\hline 15 & Vitamin D receptor & VDR & $\mathrm{P} 11473$ \\
\hline 16 & Glucose-6-phosphate 1-dehydrogenase & G6PD & P11413 \\
\hline 17 & Peroxisome proliferator-activated receptor alpha & PPARA & Q07869 \\
\hline 18 & Peroxisome proliferator-activated receptor delta & PPARD & Q03181 \\
\hline 19 & Peroxisome proliferator-activated receptor gamma & PPARG & P37231 \\
\hline 20 & UDP-glucuronosyltransferase 2B7 & UGT2B7 & P16662 \\
\hline 21 & 11-beta-hydroxysteroid dehydrogenase 2 & HSD11B2 & P80365 \\
\hline 22 & NADPH oxidase 4 & NOX4 & Q9NPH5 \\
\hline 23 & Tyrosine-protein kinase SYK & SYK & P43405 \\
\hline 24 & Glycogen synthase kinase- 3 beta & GSK3B & P49841 \\
\hline 25 & Matrix metalloproteinase 9 & MMP9 & P14780 \\
\hline 26 & Matrix metalloproteinase 2 & MMP2 & P08253 \\
\hline 27 & Matrix metalloproteinase 12 & MMP12 & P39900 \\
\hline 28 & ATP-binding cassette sub-family $G$ member 2 & ABCG2 & Q9UNQ0 \\
\hline 29 & P-glycoprotein 1 & $\mathrm{ABCB} 1$ & P08183 \\
\hline 30 & Arachidonate 12-lipoxygenase & ALOX12 & P18054 \\
\hline 31 & Cyclooxygenase- 2 & PTGS2 & P35354 \\
\hline 32 & Insulin-like growth factor I receptor & IGF1R & P08069 \\
\hline 33 & Myeloperoxidase & MPO & P05164 \\
\hline 34 & Matrix metalloproteinase 3 & MMP3 & P08254 \\
\hline 35 & Serine/threonine-protein kinase AKT & AKT1 & P31749 \\
\hline 36 & Beta-secretase 1 & BACE1 & P56817 \\
\hline 37 & Tyrosine-protein kinase receptor UFO & AXL & P30530 \\
\hline 38 & NUAK family SNF1-like kinase 1 & NUAK1 & 060285 \\
\hline 39 & Aldehyde reductase (by homology) & AKR1A1 & P14550 \\
\hline 40 & Plasminogen & PLG & P00747 \\
\hline 41 & PI3-kinase p110-delta subunit & PIK3CD & O00329 \\
\hline 42 & PI3-kinase p110-gamma subunit & PIK3CG & P48736 \\
\hline 43 & Hematopoietic prostaglandin D synthase & HPGDS & O60760 \\
\hline 44 & Serine-protein kinase ATM & ATM & Q13315 \\
\hline 45 & Cytochrome P450 24A1 & CYP24A1 & Q07973 \\
\hline 46 & Mineralocorticoid receptor & NR3C2 & P08235 \\
\hline 47 & Cannabinoid receptor 1 & CNR1 & P21554 \\
\hline 48 & Hepatocyte nuclear factor 4 -alpha & HNF4A & P41235 \\
\hline 49 & C-C chemokine receptor type 1 & CCR1 & P32246 \\
\hline 50 & Histone-lysine N-methyltransferase EZH2 & EZH2 & Q15910 \\
\hline 51 & MAP kinase p38 alpha & MAPK14 & Q16539 \\
\hline 52 & Bromodomain-containing protein 2 & BRD2 & P25440 \\
\hline 53 & Aldehyde dehydrogenase & ALDH2 & P05091 \\
\hline 54 & Fatty acid binding protein adipocyte & FABP4 & P15090 \\
\hline 55 & Fatty acid-binding protein, liver & FABP1 & P07148 \\
\hline 56 & Acyl-CoA desaturase & SCD & O00767 \\
\hline 57 & MAP kinase ERK1 & MAPK3 & P27361 \\
\hline 58 & Short transient receptor potential channel 6 & TRPC6 & Q9Y210 \\
\hline 59 & Mitogen-activated protein kinase kinase kinase 5 & MAP3K5 & Q99683 \\
\hline 60 & Disintegrin and metalloproteinase domain-containing protein 17 & ADAM17 & P78536 \\
\hline
\end{tabular}


TABLE 2: Continued.

\begin{tabular}{|c|c|c|c|}
\hline No. & Target & Common name & Uniprot ID \\
\hline 61 & Hexokinase type IV & GCK & P35557 \\
\hline 62 & Intercellular adhesion molecule-1 & ICAM1 & P05362 \\
\hline 63 & P-selectin & SELP & P16109 \\
\hline 64 & Leukocyte adhesion molecule-1 & SELL & P14151 \\
\hline 65 & Matrix metalloproteinase 1 & MMP1 & P03956 \\
\hline 66 & Matrix metalloproteinase 8 & MMP8 & P22894 \\
\hline 67 & Endothelin-converting enzyme 1 & ECE1 & P42892 \\
\hline 68 & Integrin beta-3 & ITGB3 & P05106 \\
\hline 69 & Phosphatidylinositol 4,5-bisphosphate 3-kinase catalytic subunit beta isoform & PIK3CB & P42338 \\
\hline 70 & Sorbitol dehydrogenase & SORD & Q00796 \\
\hline 71 & MAP kinase ERK2 & MAPK1 & P28482 \\
\hline 72 & Vascular endothelial growth factor receptor 1 & FLT1 & P17948 \\
\hline 73 & Matrix metalloproteinase 7 & MMP7 & P09237 \\
\hline 74 & Type- 1 angiotensin II receptor & AGTR1 & P30556 \\
\hline 75 & Glucose transporter & SLC2A1 & P11166 \\
\hline 76 & Nerve growth factor receptor Trk-A & NTRK1 & P04629 \\
\hline 77 & Tyrosine-protein kinase JAK1 & JAK1 & P23458 \\
\hline 78 & Tyrosine-protein kinase JAK2 & JAK2 & O60674 \\
\hline 79 & Sodium/glucose cotransporter 2 & SLC5A2 & P31639 \\
\hline 80 & Serine/threonine-protein kinase receptor R3 & ACVRL1 & P37023 \\
\hline 81 & Epoxide hydratase & EPHX2 & P34913 \\
\hline 82 & Cytochrome P450 11B2 & CYP11B2 & P19099 \\
\hline 83 & Endothelin receptor ET-A & EDNRA & P25101 \\
\hline 84 & Glutathione S-transferase $\mathrm{Mu} 1$ & GSTM1 & P09488 \\
\hline 85 & Interleukin-1 beta & ILIB & P01584 \\
\hline 86 & Insulin receptor & INSR & P06213 \\
\hline 87 & Protein tyrosine kinase 2 beta & PTK2B & Q14289 \\
\hline 88 & Cyclooxygenase-1 & PTGS1 & P23219 \\
\hline 89 & Cytochrome P450 2C9 & CYP2C9 & $\mathrm{P} 11712$ \\
\hline 90 & Cytochrome P450 3A4 & CYP3A4 & P08684 \\
\hline 91 & Trypsin I & PRSS1 & P07477 \\
\hline 92 & C-C chemokine receptor type 5 & CCR5 & P51681 \\
\hline 93 & Dopamine D2 receptor & DRD2 & P14416 \\
\hline 94 & Cholesteryl ester transfer protein & CETP & P11597 \\
\hline 95 & Calcitonin gene-related peptide type 1 receptor & CALCRL & Q16602 \\
\hline 96 & Serotonin $2 \mathrm{a}(5-\mathrm{HT} 2 \mathrm{a})$ receptor & HTR2A & P28223 \\
\hline 97 & Disintegrin and metalloproteinase domain-containing protein 10 & ADAM10 & O14672 \\
\hline 98 & TGF-beta receptor type I & TGFBR1 & P36897 \\
\hline 99 & Nitric-oxide synthase, brain & NOS1 & P29475 \\
\hline 100 & Cathepsin (B and $\mathrm{K})$ & CTSB & P07858 \\
\hline 101 & Bradykinin B1 receptor & BDKRB1 & P46663 \\
\hline 102 & Potassium voltage-gated channel subfamily KQT member 1 & KCNQ1 & P51787 \\
\hline 103 & Leukotriene A4 hydrolase & LTA4H & P09960 \\
\hline 104 & Apoptosis regulator $\mathrm{Bcl}-2$ & BCL2 & P10415 \\
\hline 105 & Kininogen-1 & KNG1 & P01042 \\
\hline 106 & Solute carrier family 22 member 2 & SLC22A2 & O15244 \\
\hline 107 & Plasma retinol-binding protein & RBP4 & P02753 \\
\hline 108 & Histone deacetylase 4 & HDAC4 & P56524 \\
\hline 109 & Dopamine D3 receptor & DRD3 & P35462 \\
\hline 110 & C-C chemokine receptor type 2 & CCR2 & P41597 \\
\hline 111 & Solute carrier family 22 member 12 & SLC22A12 & Q96S37 \\
\hline 112 & Glucagon-like peptide 1 receptor & GLP1R & P43220 \\
\hline 113 & Dual specificity mitogen-activated protein kinase kinase 2 & MAP2K2 & P36507 \\
\hline 114 & Death-associated protein kinase 2 & DAPK2 & Q9UIK4 \\
\hline 115 & Bile acid receptor FXR & NR1H4 & Q96RI1 \\
\hline 116 & Interleukin-6 & IL6 & P05231 \\
\hline 117 & Transcription factor AP-1 & JUN & P05412 \\
\hline 118 & Vascular endothelial growth factor A & VEGFA & P15692 \\
\hline 119 & Interleukin-10 & IL10 & P22301 \\
\hline 120 & Endothelin-1 & EDN1 & P05305 \\
\hline
\end{tabular}


TABle 2: Continued.

\begin{tabular}{|c|c|c|c|}
\hline No. & Target & Common name & Uniprot ID \\
\hline 121 & Nitric oxide synthase, endothelial & NOS3 & P29474 \\
\hline 122 & Urotensin II receptor & UTS2R & Q9UKP6 \\
\hline 123 & $78 \mathrm{kDa}$ glucose-regulated protein & HSPA5 & P11021 \\
\hline 124 & Galectin-3 & LGALS3 & P17931 \\
\hline 125 & Macrophage migration inhibitory factor & MIF & P14174 \\
\hline 126 & Serum paraoxonase/arylesterase 1 & PON1 & P27169 \\
\hline 127 & Kallikrein 1 & KLK1 & P06870 \\
\hline 128 & Rho-associated protein kinase 1 & ROCK1 & Q13464 \\
\hline 129 & Sphingosine kinase 1 & SPHK1 & Q9NYA1 \\
\hline 130 & Serine/threonine-protein kinase Sgk1 & SGK1 & O00141 \\
\hline 131 & Low affinity sodium-glucose cotransporter & SLC5A4 & Q9NY91 \\
\hline 132 & Neutrophil cytosol factor 1 & NCF1 & P14598 \\
\hline 133 & Antileukoproteinase & SLPI & P03973 \\
\hline 134 & Signal transducer and activator of transcription 1-alpha/beta & STAT1 & P42224 \\
\hline 135 & Protein kinase $\mathrm{C}$ beta type & PRKCB & P05771 \\
\hline 136 & Gap junction alpha-1 protein & GJA1 & P17302 \\
\hline 137 & C-X-C motif chemokine 11 & CXCL11 & $\mathrm{O} 14625$ \\
\hline 138 & Interleukin-8 & CXCL8 & P10145 \\
\hline 139 & Superoxide dismutase $[\mathrm{Cu}-\mathrm{Zn}]$ & SOD1 & P00441 \\
\hline 140 & C-C motif chemokine 2 & CCL2 & P13500 \\
\hline 141 & Hypoxia-inducible factor 1-alpha & HIF1A & Q16665 \\
\hline 142 & Caveolin-1 & CAV1 & Q03135 \\
\hline 143 & Interleukin-1 alpha & IL1A & P01583 \\
\hline 144 & Nuclear factor erythroid 2-related factor 2 & NFE2L2 & Q16236 \\
\hline 145 & C-X-C motif chemokine 10 & CXCL10 & P02778 \\
\hline 146 & Plasminogen activator inhibitor 1 & SERPINE1 & P05121 \\
\hline 147 & Osteopontin & SPP1 & P10451 \\
\hline 148 & Bone morphogenetic protein 2 & BMP2 & P12643 \\
\hline 149 & Transforming growth factor beta- 1 proprotein & TGFB1 & $\mathrm{P} 01137$ \\
\hline 150 & Cyclin-dependent kinase inhibitor $2 \mathrm{~A}$ & CDKN2A & P42771 \\
\hline 151 & Transcription factor E2F1 & E2F1 & Q01094 \\
\hline 152 & Thrombomodulin & THBD & P07204 \\
\hline 153 & Insulin-like growth factor II & IGF2 & $\mathrm{P} 01344$ \\
\hline 154 & Catalase & CAT & P04040 \\
\hline 155 & $\begin{array}{l}\text { Phosphatidylinositol 3,4,5-trisphosphate 3-phosphatase and } \\
\text { dual-specificity protein phosphatase PTEN }\end{array}$ & PTEN & P60484 \\
\hline 156 & Pro-epidermal growth factor & EGF & P01133 \\
\hline 157 & ATP synthase subunit beta, mitochondria & ATP5F1B & P06576 \\
\hline 158 & NAD-dependent protein deacetylase sirtuin-1 & SIRT1 & Q96EB6 \\
\hline 159 & Angiotensin-converting enzyme & $\mathrm{ACE}$ & P12821 \\
\hline 160 & Matrix metalloproteinase 10 & MMP10 & P09238 \\
\hline 161 & Transketolase & TKT & P29401 \\
\hline 162 & Dipeptidyl peptidase IV & DPP4 & P27487 \\
\hline 163 & Nuclear factor NF-kappa-B p65 subunit & RELA & Q04206 \\
\hline 164 & Nitric oxide synthase, inducible & NOS2 & P35228 \\
\hline 165 & Protein kinase $\mathrm{C}$ alpha type & PRKCA & P17252 \\
\hline 166 & Tumor necrosis factor & TNF & $\mathrm{P} 01375$ \\
\hline 167 & Protein kinase $\mathrm{C}$ epsilon type & PRKCE & Q02156 \\
\hline 168 & Renin & REN & P00797 \\
\hline 169 & Axin1/beta-catenin & CTNNB1 & P35222 \\
\hline 170 & Fibronectin & FN1 & P02751 \\
\hline 171 & C-X-C chemokine receptor type 4 & CXCR4 & P61073 \\
\hline 172 & Heparanase & HPSE & Q9Y251 \\
\hline 173 & Glucagon & GCG & $\mathrm{P} 01275$ \\
\hline 174 & Tumor necrosis factor receptor superfamily member $11 \mathrm{~B}$ & TNFRSF11B & O00300 \\
\hline 175 & Metalloproteinase inhibitor 1 & TIMP1 & P01033 \\
\hline 176 & Metalloproteinase inhibitor 2 & TIMP2 & P16035 \\
\hline 177 & Fibroblast growth factor 2 & FGF2 & P09038 \\
\hline 178 & Lipoprotein lipase & LPL & P06858 \\
\hline 179 & Coagulation factor $\mathrm{V}$ & F5 & P12259 \\
\hline
\end{tabular}


TABLE 2: Continued.

\begin{tabular}{lccc}
\hline No. & Target & Common name & Uniprot ID \\
\hline 180 & Cyclic AMP-responsive element-binding protein 1 & CREB1 & P16220 \\
181 & Phosphatidylinositol 3,4,5-trisphosphate 5- phosphatase 2 & INPPL1 & O15357 \\
182 & Tumor necrosis factor ligand superfamily member 6 & FASLG & P48023 \\
\hline
\end{tabular}

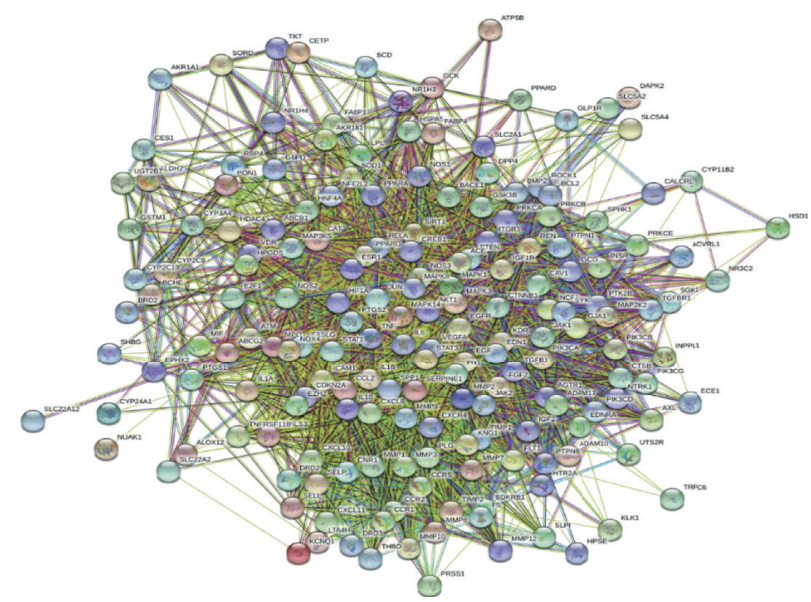

Figure 1: PPI network of the 182 common targets.

TABLE 3: Thirty key targets obtained by network topology analysis.

\begin{tabular}{|c|c|c|c|c|}
\hline Serial number & Node & Degree & Closeness centrality & Betweenness centrality \\
\hline 1 & PIK3CA & 40 & 0.49508197 & 0.09370214 \\
\hline 2 & STAT3 & 40 & 0.5 & 0.0863086 \\
\hline 3 & AKT1 & 35 & 0.49025974 & 0.15311921 \\
\hline 4 & KNG1 & 33 & 0.44023324 & 0.06128185 \\
\hline 5 & VEGFA & 33 & 0.49185668 & 0.06953442 \\
\hline 6 & JUN & 32 & 0.48089172 & 0.07229449 \\
\hline 7 & MAPK3 & 30 & 0.4617737 & 0.02240476 \\
\hline 8 & MAPK1 & 30 & 0.4689441 & 0.06714477 \\
\hline 9 & EGF & 27 & 0.4617737 & 0.0336672 \\
\hline 10 & EDN1 & 27 & 0.46604938 & 0.05180077 \\
\hline 11 & EGFR & 26 & 0.44023324 & 0.01794429 \\
\hline 12 & JAK1 & 26 & 0.44940476 & 0.02254905 \\
\hline 13 & IL6 & 26 & 0.45209581 & 0.02532622 \\
\hline 14 & CXCL8 & 25 & 0.43768116 & 0.03191743 \\
\hline 15 & RELA & 24 & 0.45757576 & 0.04035241 \\
\hline 16 & FN1 & 23 & 0.4351585 & 0.01464828 \\
\hline 17 & JAK2 & 23 & 0.44940476 & 0.01620852 \\
\hline 18 & CTNNB1 & 23 & 0.45481928 & 0.06488997 \\
\hline 19 & TNF & 22 & 0.44281525 & 0.0272631 \\
\hline 20 & TGFB1 & 21 & 0.44281525 & 0.03270724 \\
\hline 21 & MMP9 & 20 & 0.40921409 & 0.03200512 \\
\hline 22 & CXCR4 & 19 & 0.41032609 & 0.01402652 \\
\hline 23 & TIMP1 & 19 & 0.41712707 & 0.00798146 \\
\hline 24 & MAPK14 & 19 & 0.44411765 & 0.01628416 \\
\hline 25 & BDKRB1 & 18 & 0.3994709 & 0.00725308 \\
\hline 26 & PIK3CB & 18 & 0.40921409 & 0.00732155 \\
\hline 27 & MAPK8 & 18 & 0.42296919 & 0.03099697 \\
\hline 28 & ITGB3 & 18 & 0.42296919 & 0.01050834 \\
\hline 29 & CCR5 & 16 & 0.39841689 & 0.00592392 \\
\hline 30 & PLG & 16 & 0.40266667 & 0.02168017 \\
\hline
\end{tabular}




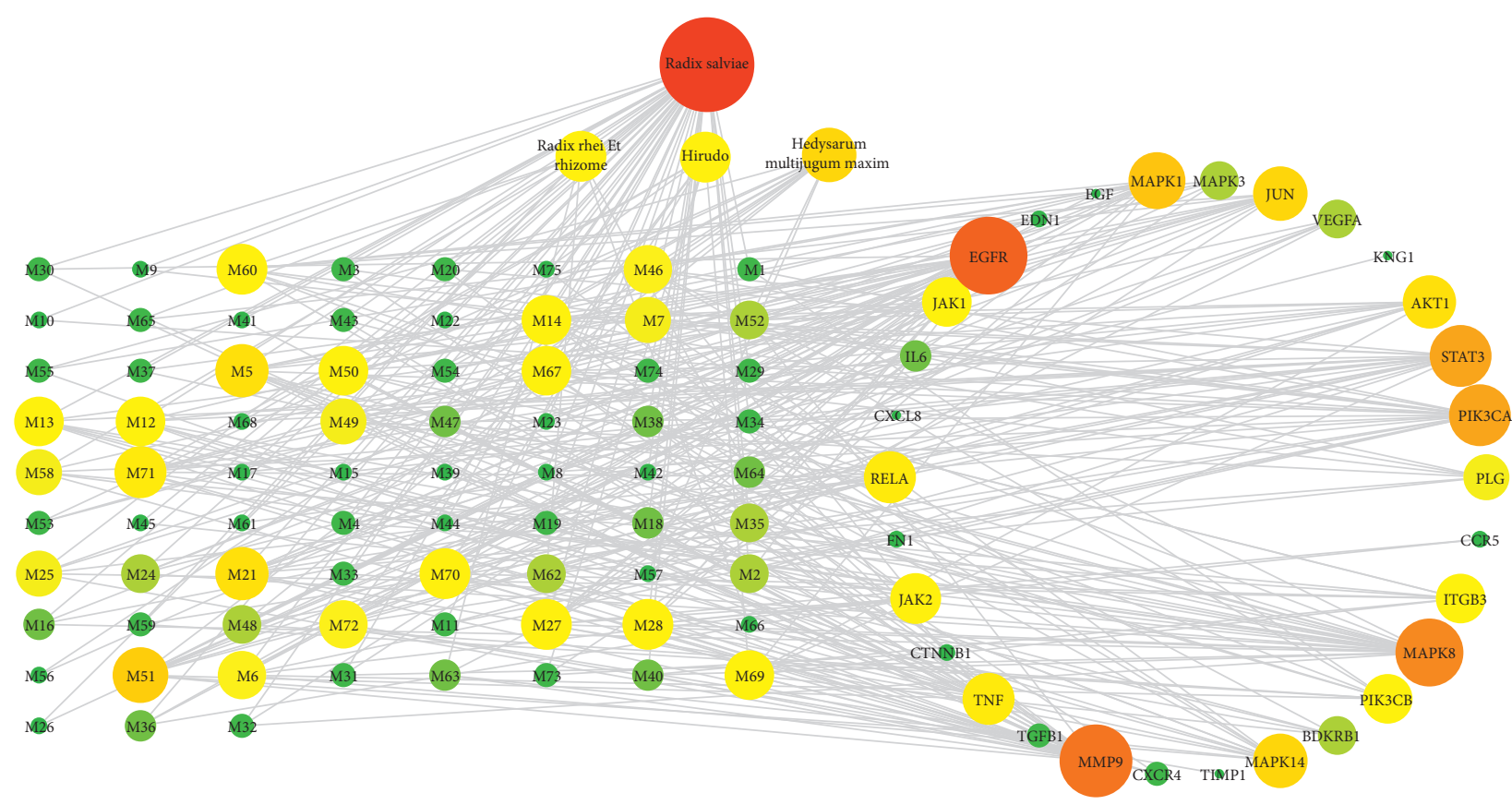

Figure 2: "SZF-key targets-DKD" network. The nodes were visualized with degree. The larger and the redder the node, the higher the degree it was. M1-75 stand for the active ingredients whose full names are shown in Table 4.

TABLE 4: The information of active ingredients.

\begin{tabular}{|c|c|c|}
\hline No. & Active ingredients & Code name \\
\hline 1 & Isoimperatorin & M1 \\
\hline 2 & 1,2,5,6-Tetrahydrotanshinone & M2 \\
\hline 3 & 5,6-Dihydroxy-7-isopropyl-1,1-dimethyl-2,3-dihydrophenanthren-4-one & M3 \\
\hline 4 & (E)-3-[2-(3,4-Dihydroxyphenyl)-7-hydroxy-benzofuran-4-yl]acrylic & M4 \\
\hline 5 & 2-(4-Hydroxy-3-methoxyphenyl)-5-(3-hydroxypropyl)-7-methoxy-3-benzofurancarboxaldehyde & M5 \\
\hline 6 & Przewaquinone $\mathrm{c}$ & M6 \\
\hline 7 & Cryptotanshinone & M7 \\
\hline 8 & Dihydrotanshinlactone & M8 \\
\hline 9 & Isotanshinone II & M9 \\
\hline 10 & Miltipolone & M10 \\
\hline 11 & Miltirone & M11 \\
\hline 12 & Tanshinaldehyde & M12 \\
\hline 13 & Danshenol B & M13 \\
\hline 14 & Danshenol A & M14 \\
\hline 15 & Deoxyneocryptotanshinone & M15 \\
\hline 16 & Dihydrotanshinone I & M16 \\
\hline 17 & Miltionone I & M17 \\
\hline 18 & Miltionone II & M18 \\
\hline 19 & Neocryptotanshinone ii & M19 \\
\hline 20 & Neocryptotanshinone & M20 \\
\hline 21 & Luteolin & M21 \\
\hline 22 & Salvilenone I & M22 \\
\hline 23 & Salviolone & M23 \\
\hline 24 & Epidanshenspiroketallactone & M24 \\
\hline 25 & Tanshinone iia & M25 \\
\hline 26 & $\alpha$-Amyrin & M26 \\
\hline 27 & Dan-shexinkum d & M27 \\
\hline 28 & Sclareol & M28 \\
\hline 29 & Dehydrotanshinone II A & M29 \\
\hline 30 & Baicalin & M30 \\
\hline 31 & 2-Isopropyl-8-methylphenanthrene-3,4-dione & M31 \\
\hline 32 & Formyltanshinone & M32 \\
\hline
\end{tabular}


TABle 4: Continued.

\begin{tabular}{|c|c|c|}
\hline No. & Active ingredients & Code name \\
\hline 33 & 3-Beta-Hydroxymethyllenetanshiquinone & M33 \\
\hline 34 & Methylenetanshinquinone & M34 \\
\hline 35 & (2R)-3-(3,4-Dihydroxyphenyl)-2-[(Z)-3-(3,4-dihydroxyphenyl)acryloyl]oxy-propionic acid & M35 \\
\hline 36 & (6S)-6-(Hydroxymethyl)-1,6-dimethyl-8,9-dihydro-7H-naphtho[8,7-g]benzofuran-10,11-dione & M36 \\
\hline 37 & Tanshinone VI & M37 \\
\hline 38 & Przewalskin b & M38 \\
\hline 39 & 6-o-Syringyl-8-o-acetyl shanzhiside methyl ester & M39 \\
\hline 40 & Prolithospermic acid & M40 \\
\hline 41 & (Z)-3-[2-[(E)-2-(3,4-Dihydroxyphenyl)vinyl]-3,4-dihydroxyphenyl]acrylic acid & M41 \\
\hline 42 & Salvianolic acid g & M42 \\
\hline 43 & Salvianolic acid $\mathrm{j}$ & M43 \\
\hline 44 & Danshenspiroketallactone & M44 \\
\hline 45 & 1-Methyl-8,9-dihydro-7H-naphtho[5,6-g]benzofuran-6,10,11-trione & M45 \\
\hline 46 & 3,9-di-O-MethylnissolinM & M46 \\
\hline 47 & (6aR,11aR)-9,10-Dimethoxy-6a,11a-dihydro-6H-benzofurano[3,2-c]chromen-3-ol & M47 \\
\hline 48 & (3R)-3-(2-Hydroxy-3,4-dimethoxyphenyl)chroman-7-ol & M48 \\
\hline 49 & Isorhamnetin & M49 \\
\hline 50 & Kaempferol & M50 \\
\hline 51 & Quercetin & M51 \\
\hline 52 & Jaranol & M52 \\
\hline 53 & Bifendate & M53 \\
\hline 54 & Formononetin & M54 \\
\hline 55 & Isoflavanone & M55 \\
\hline 56 & Calycosin & M56 \\
\hline 57 & Hederagenin & M57 \\
\hline 58 & Sennoside E_qt & M58 \\
\hline 59 & Toralactone & M59 \\
\hline 60 & Palmidin A & M60 \\
\hline 61 & Daucosterol_qt & M61 \\
\hline 62 & Eupatin & M62 \\
\hline 63 & Procyanidin B-5,3'-O-gallate & M63 \\
\hline 64 & Rhein & M64 \\
\hline 65 & Beta-sitosterol & M65 \\
\hline 66 & Aloe-emodin & M66 \\
\hline 67 & Lipase & M67 \\
\hline 68 & Gardnerilin a & M68 \\
\hline 69 & Hirudin & M69 \\
\hline 70 & o-Desulfated heparin & M70 \\
\hline 71 & Ursolic acid & M71 \\
\hline 72 & Heparin & M72 \\
\hline 73 & Genioisidic acid & M73 \\
\hline 74 & Genipinic acid & M74 \\
\hline 75 & Nadroparin & M75 \\
\hline
\end{tabular}

transcription from RNA polymerase II promoter, inflammatory response, lipopolysaccharide-mediated signaling pathway, positive regulation of peptidyl-serine phosphorylation, and other biological processes. As the top $20 \mathrm{GO}$ enrichment items listed, DKD is relevant to kinds of $\mathrm{BP}$ in body abnormalities, and SZF is likely to regulate these items and then play an anti-DKD role.

KEGG pathway enrichment analysis showed that a total of 104 pathways were obtained. The top 20 pathways are displayed in Figure 4, which include TNF signaling pathway, HIF-1 signaling pathway, Toll-like receptor signaling pathway, FoxO signaling pathway, NOD-like receptor signaling pathway, and so on.

\section{Discussion}

Previous studies have suggested that SZF has a therapeutic effect on DKD $[5,6]$. However, the potential mechanisms of SZF treating in DKD have not been fully explained. In this study, we mainly applied network pharmacology to explore it. Firstly, a total of 140 potential active compounds and 182 common targets of SZF and DKD were obtained after screening of active compounds and mapping of targets. Then, we constructed two networks, including the PPI network of 182 common targets and SZF-key targets-DKD network, and then applying GO and KEGG enrichment analysis to explore the regulation mechanism of SZF in treating $\mathrm{DKD}$. 


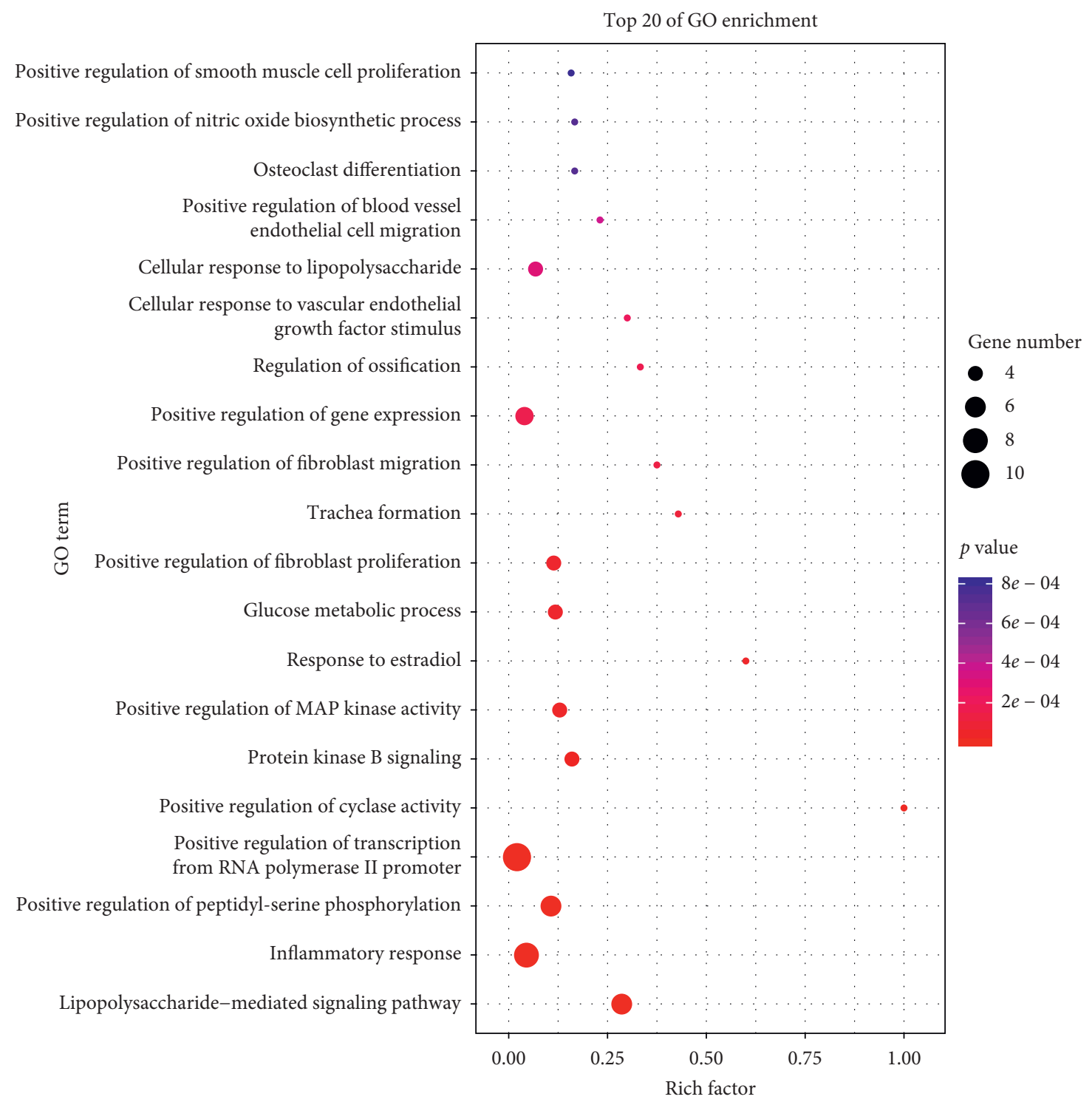

FIgURE 3: Top 20 enrichments in GO analysis.

Through the SZF-key targets-DKD network, we could know that most active ingredients were linked with no less than one target, which indicated the character of multitarget of TCM active ingredients. In the meanwhile, different active compounds from different herbs acted on the same targets, which demonstrated that SZF had a synergistic effect in treating DKD. In addition, there were 8 active ingredients whose degrees were greater than 2 times of average in SZFkey targets-DKD network topology analysis. Interestingly, 3 of them had been proven to have kidney protection effect by experiments. For example, quercetin liposomes had renal protective effects of reducing oxidative stress, attenuating AGE expression, and delaying the progression of DKD [26]. Luteolin attenuated DKD mainly via suppression of inflammatory response and oxidative response [27]. Ursolic acid alleviated renal damage in type 2 diabetic $\mathrm{db} / \mathrm{db}$ mice by downregulating proteins in the angiotensin II type 1 receptor-associated protein/angiotensin II type 1 receptor signaling pathway to inhibit extracellular matrix accumulation, renal inflammation, fibrosis, and oxidative stress [28]. These results were coincident with our predictions, which suggested that active ingredients with higher degree might play an important role in the treatment of DKD. Meanwhile, we discovered five active ingredients (M5, M27, M28, M60, and M70) that were likely to have renal protection effect but had not been verified up to now.

Moreover, the results of the SZF-key targets-DKD topology analysis also showed that there were 5 targets whose degrees were greater than 2 times of the average. Particularly, 3 of these had been proven to be closely related with DKD. For instance, EGFR activation had a significant role in activating pathways that mediate podocyte injury and loss in diabetic nephropathy [29]. Downregulated expression of MMP-9 could promote the process of DKD [30]. STAT3 inhibition could hinder the development and progression of DKD in diabetic patients [31]. 


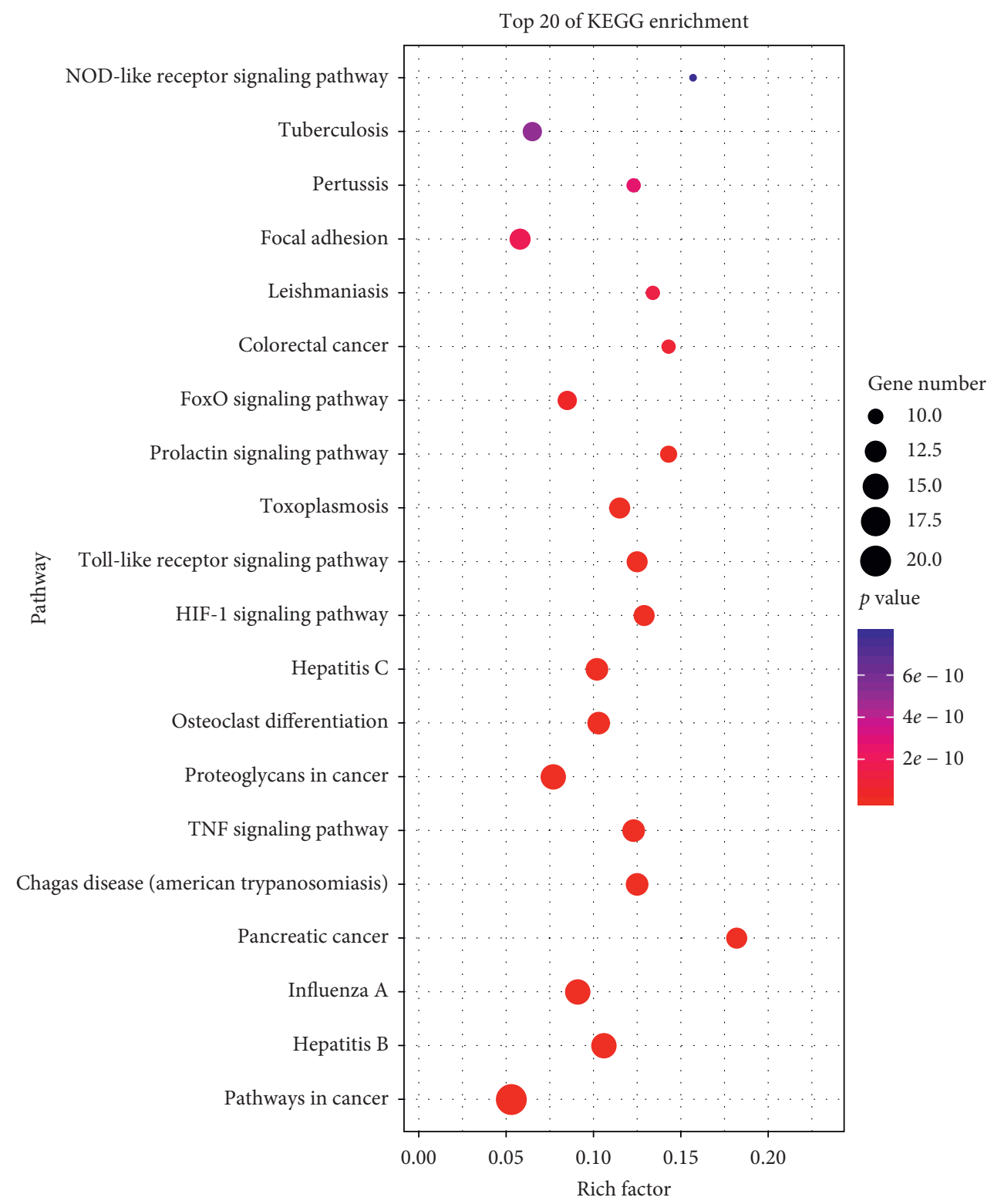

FIgURE 4: Top 20 KEGG pathway enrichments.

As shown in GO analysis, the potential targets of SZF acting on DKD were mainly associated with various biological processes, such as lipopolysaccharide-mediated signaling pathway, inflammatory response, positive regulation of cyclase activity, protein kinase B signaling, positive regulation of MAP kinase activity, and response to estradiol, which had a strongly direct correlation with the pathogenesis of DKD [32-38].

Similarly, KEGG pathway enrichment analysis showed that SZF took an anti-DKD effect by multiple pathways. Through further research, we found that some pathways had been already verified to exert anti-DKD potential by experiments, such as TNF signaling pathway [39], HIF-1 signaling pathway [40], Toll-like receptor signaling pathway [41], FoxO signaling pathway [42], focal adhesion [43], and NOD-like receptor signaling pathway [44]. These results were also consistent with what we predicted.
In addition, SZF might have potential therapeutic effects on diseases such as cancer, hepatitis, influenza, leishmaniasis, pertussis, and tuberculosis according to the KEGG enrichment analysis. Just as it was reported that different diseases had common or similar pathological changes and could be treated with the same prescription [45], the above results suggested that SZF concentrated more on the systematicness of the body when treating DKD. In other words, SZF possibly regulated the body to reach the balance state, then reaching the aim of treatment.

\section{Conclusion}

In conclusion, this study based on the network pharmacology had preliminarily explained the anti-DKD mechanism of SZF from the perspective of multi-active ingredients, multi-targets, 
and multi-pathway. In the future, we will further investigate its mechanism by molecular docking, using in vitro or in vivo studies.

\section{Data Availability}

The data used to support the results of this study can be obtained from the corresponding author upon reasonable request.

\section{Conflicts of Interest}

The authors declare that there are no conflicts of interest.

\section{Authors' Contributions}

Wang Xin-miao, Yang Hao-yu, and Zhang Li-li contributed equally to this work.

\section{Acknowledgments}

This study was supported by the Beijing Natural Science Foundation (7212189), the Fundamental Research Funds for the Central Public Welfare Research Institutes (ZZ13-ZD06), and the Fundamental Research Funds for the China Academy of Chinese Medical Sciences (ZZ0808004).

\section{References}

[1] A.-L. Cao, L. Wang, X. Chen et al., "Ursodeoxycholic acid and 4-phenylbutyrate prevent endoplasmic reticulum stress-induced podocyte apoptosis in diabetic nephropathy," Laboratory Investigation, vol. 96, no. 6, pp. 610-622, 2016.

[2] G.-D. Sun, W.-P. Cui, Q.-Y. Guo, and L.-N. Miao, "Histone lysine methylation in diabetic nephropathy," Journal of Diabetes Research, vol. 2014, Article ID 654148, 9 pages, 2014.

[3] R. Z. Alicic, M. T. Rooney, and K. R. Tuttle, "Diabetic kidney disease," Clinical Journal of the American Society of $\mathrm{Ne}$ phrology, vol. 12, no. 12, pp. 2032-2045, 2017.

[4] C. Magee, D. J. Grieve, C. J. Watson, and D. P. Brazil, "Diabetic nephropathy: a tangled web to unweave," Cardiovascular Drugs and Therapy, vol. 31, no. 5-6, pp. 579-592, 2017.

[5] H. Chen, J. Guo, X. Zhao et al., "Retrospective analysis of the overt proteinuria diabetic kidney disease in the treatment of modified Shenzhuo formula for 2 years," Medicine (Baltimore), vol. 96, no. 12, p. e6349, 2017.

[6] J. Tian, L. Zhao, Q. Zhou et al., "Retrospective analysis on modified Didang Tang for treating microalbuminuria of diabetic nephropathy," Journal of Beijing University of Traditional Chinese Medicine, vol. 19, no. 6, pp. 7-10, 2012.

[7] Y. Y. Jiang, M. Q. Sun, B. Ma et al., "The application and thinking of omics technologies in the modern pharmacology research of traditional Chinese medine," World Science and Technology/Modernization of Traditional Chinese Medicine and Materia Medica, vol. 20, no. 8, pp. 1287-1295, 2018.

[8] M. Isgut, M. Rao, C. Yang, V. Subrahmanyam, P. C. G. Rida, and R. Aneja, "Application of combination high-throughput phenotypic screening and target identification methods for the discovery of natural product-based combination drugs," Medicinal Research Reviews, vol. 38, no. 2, pp. 504-524, 2018.

[9] G.-B. Zhang, Q.-Y. Li, Q.-L. Chen, and S.-B. Su, "Network pharmacology: a new approach for Chinese herbal medicine research," Evidence-based Complementary and Alternative Medicine, vol. 2013, Article ID 621423, 9 pages, 2013.

[10] A. L. Hopkins, "Network pharmacology: the next paradigm in drug discovery," Nature Chemical Biology, vol. 4, no. 11, pp. 682-690, 2008.

[11] J. Ru, P. Li, J. Wang et al., "TCMSP: a database of systems pharmacology for drug discovery from herbal medicines," Journal of Cheminformatics, vol. 6, p. 13, 2014.

[12] R. Xue, Z. Fang, M. Zhang, Z. Yi, C. Wen, and T. Shi, "TCMID: traditional Chinese Medicine integrative database for herb molecular mechanism analysis," Nucleic Acids Research, vol. 41, no. D1, pp. D1089-D1095, 2013.

[13] A. Daina, O. Michielin, and V. Zoete, "SwissTargetPrediction: updated data and new features for efficient prediction of protein targets of small molecules," Nucleic Acids Research, vol. 47, no. W1, pp. W357-W364, 2019.

[14] H. Ye, L. Ye, H. Kang et al., "HIT: linking herbal active ingredients to targets," Nucleic Acids Research, vol. 39, no. suppl_1, pp. D1055-D1059, 2011.

[15] Y. H. Li, C. Y. Yu, X. X. Li et al., “Therapeutic target database update 2018: enriched resource for facilitating bench-to-clinic research of targeted therapeutics," Nucleic Acids Research, vol. 46, no. D1, pp. D1121-D1127, 2018.

[16] D. S. Wishart, Y. D. Feunang, A. C. Guo et al., "DrugBank 5.0: a major update to the DrugBank database for 2018," Nucleic Acids Research, vol. 46, no. D1, pp. D1074-D1082, 2018.

[17] J. Piñero, À. Bravo, N. Queralt-Rosinach et al., "DisGeNET: a comprehensive platform integrating information on human disease-associated genes and variants," Nucleic Acids Research, vol. 45, no. D1, pp. D833-D839, 2017.

[18] D. Szklarczyk, A. L. Gable, D. Lyon et al., "STRING v11: protein-protein association networks with increased coverage, supporting functional discovery in genome-wide experimental datasets," Nucleic Acids Research, vol. 47, no. D1, pp. D607-D613, 2019.

[19] P. Shannon, A. Markiel, O. Ozier et al., "Cytoscape: a software environment for integrated models of biomolecular interaction networks," Genome Research, vol. 13, no. 11, pp. 2498-2504, 2003.

[20] D. W. Huang, B. T. Sherman, and R. A. Lempicki, "Systematic and integrative analysis of large gene lists using DAVID bioinformatics resources," Nature Protocols, vol. 4, no. 1, pp. 44-57, 2009.

[21] M. Ashburner, C. A. Ball, J. A. Blake et al., "Gene ontology: tool for the unification of biology," Nature Genetics, vol. 25, no. 1, pp. 25-29, 2000.

[22] L. Zhou, Z. Liu, Z Wang et al., "Astragalus polysaccharides exerts immunomodulatory effects via TLR4-mediated MyD88-dependent signaling pathway in vitro and in vivo," Scientific Reports, vol. 7, p. 44822, 2017.

[23] L. Li, X. Hou, R. Xu, C. Liu, and M. Tu, "Research review on the pharmacological effects of astragaloside IV," Fundamental and Clinical Pharmacology, vol. 31, no. 1, pp. 17-36, 2017.

[24] X.-Y. Bao, Q. Zheng, Q. Tong et al., "Danshensu for myocardial ischemic injury: precli-nical evidence and novel methodology of quality assessment tool," Frontiers in Pharmacology, vol. 9, p. 1445, 2018.

[25] X. Dong, J. Fu, X. Yin et al., "Emodin: a review of its pharmacology, toxicity and pharmacokinetics," Phytotherapy Research, vol. 30, no. 8, pp. 1217-1218, 2016.

[26] L. Tang, K. Li, Y. Zhang et al., "Quercetin liposomes ameliorate streptozotocin-induced diabetic nephropathy in diabetic rats," Scientific Reports, vol. 10, no. 1, p. 2440, 2020. 
[27] M. Zhang, L. He, J. Liu, and L. Zhou, "Luteolin attenuates diabetic nephropathy through suppressing inflammatory response and oxidative stress by inhibiting STAT3 pathway," Experimental and Clinical Endocrinology and Diabetes, vol. 128, 2020.

[28] T. Ma, L. Xu, L. Lu et al., "Ursolic acid treatment alleviates diabetic kidney injury by regulating the ARAP1/AT1R signaling pathway," Diabetes, Metabolic Syndrome and Obesity: Targets and Therapy, vol. 12, pp. 2597-2608, 2019.

[29] J. Chen, J.-K. Chen, and R. C. Harris, "EGF receptor deletion in podocytes attenuates diabetic nephropathy," Journal of the American Society of Nephrology, vol. 26, no. 5, pp. 1115-1125, 2015.

[30] L. Liu and J Tan, "The relationship between TIMP-1, MMP-9 and diabetic nephropathy," Chinese Journal of Clinical Rational Drug Use, vol. 7, no. 29, pp. 173-174, 2014.

[31] E. Said, A. Z. Sawsan, M. Eldosoky, and N. M. Elsherbeny, "Nifuroxazide, a STAT3 inhibitor, mitigates inflammatory burden and protects against diabetes-induced nephropathy in rats," Chemico-Biological Interactions, vol. 281, pp. 111-120, 2018.

[32] W. Huang, F. Guo, Y. Long et al., "High glucose and lipopolysaccharide activate NOD1- RICK-NF-kappaB inflammatory signaling in mesangial cells," Experimental and Clinical Endocrinology and Diabetes, vol. 124, no. 8, pp. 512-517, 2016.

[33] J. Donate-Correa, D. Luis-Rodríguez, E. Martín-Núñez et al., "Inflammatory targets in diabetic nephropathy," Journal of Clinical Medicine, vol. 9, no. 2, p. 458, 2020.

[34] S. Xiao, Q. Li, L. Hu et al., "Soluble guanylate cyclase stimulators and activators: where are we and where to go?," MiniReviews in Medicinal Chemistry, vol. 19, no. 18, pp. 1544-1557, 2019.

[35] K. Sakamoto, K. Kuno, M. Takemoto et al., "Pituitary adenylate cyclase-activating polypeptide protects glomerular podocytes from inflammatory injuries," Journal of Diabetes Research, vol. 2015, Article ID 727152, 10 pages, 2015.

[36] G. Wang, Y. Yan, N. Xu, Y. Hui, and D. Yin, "Upregulation of microRNA-424 relieved diabetic nephropathy by targeting Rictor through mTOR complex2/protein kinase B signaling," Journal of Cellular Physiology, vol. 234, no. 7, pp. 1164611653, 2019.

[37] R.-M. Wang, Z.-B. Wang, Y. Wang et al., "Swiprosin-1 promotes mitochondria-dependent apoptosis of glomerular podocytes via P38 MAPK pathway in early-stage diabetic nephropathy," Cellular Physiology and Biochemistry, vol. 45, no. 3, pp. 899-916, 2018.

[38] A. Inada, O. Inada, N. L. Fujii et al., “Adjusting the $17 \beta$ Estradiol-to-Androgen ratio ameliorates diabetic nephropathy," Journal of the American Society of Nephrology, vol. 27, no. 10, pp. 3035-3050, 2016.

[39] K. Omote, T. Gohda, M. Murakoshi et al., "Role of the TNF pathway in the progression of diabetic nephropathy in KK-Ay mice," American Journal of Physiology-Renal Physiology, vol. 306, no. 11, pp. F1335-F1347, 2014.

[40] R. Bohuslavova, R. Cerychova, K. Nepomucka, and G. Pavlinkova, "Renal injury is accelerated by global hypoxiainducible factor 1 alpha deficiency in a mouse model of STZinduced diabetes," BMC Endocrine Disorders, vol. 17, no. 1, p. 48, 2017.

[41] X. Y. Wu, J. Yu, and H. M Tian, "Effect of SOCS1 on diabetic renal injury through regulating TLR signaling pathway," European Review for Medical and Pharmacological Sciences, vol. 23, no. 18, pp. 8068-8074, 2019.
[42] Y. A. Hong, J. H. Lim, M. Y. Kim et al., "Extracellular superoxide dismutase attenuates renal oxidative stress through the activation of adenosine monophosphate-activated protein kinase in diabetic nephropathy," Antioxidants \& Redox Signaling, vol. 28, no. 17, pp. 1543-1561, 2018.

[43] R. Yan, Y. Wang, M. Shi et al., "Regulation of PTEN/AKT/ FAK pathways by PPARgamma impacts on fibrosis in diabetic nephropathy," Journal of Cellular Biochemistry, vol. 120, 2019.

[44] P. Luan, J. Zhuang, J. Zou et al., "NLRC5 deficiency ameliorates diabetic nephropathy through alleviating inflammation," FASEB Journal: Official Publication of the Federation of American Societies for Experimental Biology, vol. 32, no. 2, pp. 1070-1084, 2018.

[45] W.-Y. Jiang, "Therapeutic wisdom in traditional Chinese medicine: a perspective from modern science," Trends in Pharmacological Sciences, vol. 26, no. 11, pp. 558-563, 2005. 